\title{
Late Cretaceous record of large soft-bodied coleoids based on lower jaw remains from Hokkaido, Japan
}

Kazushige Tanabe, Akihiro Misaki, and Takao Ubukata

Acta Palaeontologica Polonica 60 (1), 2015: 27-38 doi:http://dx.doi.org/10.4202/app.00052.2013

The origin and phylogenetic relationships of most modern coleoid groups have not yet been explained by reliable fossil evidence, in large part because of the reduction or disappearance of a calcified chambered shell during their evolutionary history. Herein we describe two exceptionally large coleoid lower jaws from the Upper Cretaceous strata in Hokkaido, Japan. On the basis of the comparison of gross morphology and morphometric data of the lower jaws of modern and fossil coleoids, we assigned the two lower jaws to the following new taxa: Nanaimoteuthis hikidai sp. nov. of the order Vampyromorpha (superorder Octobrachia) and Haboroteuthis poseidon gen. et sp. nov. of the order Teuthida (superorder Decabrachia). The lower jaw of $N$. hikidai is distinguished from other species of the same genus from the Upper Cretaceous of Vancouver Island (Canada) and Hokkaido by having a broader, more anteriorly curved hood of the outer lamella. The lower jaw of $H$. poseidon seemingly exhibits mosaic features like those of modern teuthids and sepiids but is assigned to Teuthida on the basis of the overall shape of the outer lamella and the development of a distinct fold on the lateral wall. Because of the unusually large lower jaws, these new taxa appear to be comparable in body size to modern giant squids (Architeuthis spp.) and the Humboldt squid (Dosidicus gigas). This and other discoveries of large jaws referable to octobrachian and decabrachian coleoids from the Upper Cretaceous strata of the North Pacific fill the gap in the relatively poor fossil record of mainly soft-bodied coleoids.

Key words: Cephalopoda, Coleoidea, Vampyromorpha, Teuthida, jaws, Cretaceous, Hokkaido, Japan.

Kazushige Tanabe [tanabe@um.u-tokyo.ac.jp], Department of Historical Geology and Palaeontology, The University Museum, The University of Tokyo, Hongo 7-3-1, Tokyo 113-0033, Japan; Akihiro Misaki [misaki_a@kmnh.jp], Kitakyushu Museum of Natural History and Human History, 2-4-1 Higashida Kitakyushu City, Fukuoka 805-0071, Japan; Takao Ubukata [sbtubuk@ipc.shizuoka.ac.jp], Institute of Geosciences, Shizuoka University, Oya 836, Surugaku, Shizuoka 422-8529, Japan. 
This is an open-access article distributed under the terms of the Creative Commons

Attribution License (for details please see creativecommons.org), which permits unrestricted use, distribution, and reproduction in any medium, provided the original author and source are credited.

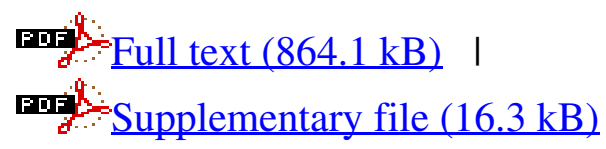

